IFUSP/P-1323/98

IFT-P.083/98

\title{
Hunting a Light $U(1)_{B}$ Gauge Boson Coupled to Baryon Number in Collider Experiments
}

\author{
M. Drees ${ }^{1 *}$, O. J. P. Éboli ${ }^{1 \dagger}$, J. K. Mizukoshi ${ }^{2 \ddagger}$ \\ ${ }^{1}$ Instituto de Física Teórica - UNESP \\ R. Pamplona 145, 01405-900 São Paulo, Brazil \\ ${ }^{2}$ Instituto de Física, Universidade de São Paulo, \\ C. P. 66.318, 05389-970 São Paulo, Brazil.
}

\begin{abstract}
We analyze several signals at HERA and the Tevatron of a light $U(1)_{B}$ gauge boson $\left(\gamma_{B}\right)$ coupling to baryon number. We show that the study of the production of $b \bar{b}$ pairs at the (upgraded) Tevatron can exclude $\gamma_{B}$ with masses $\left(m_{B}\right)$ in the range $40 \lesssim m_{B} \lesssim 300 \mathrm{GeV}$ for $\gamma_{B}$ couplings $\left(\alpha_{B}\right)$ greater than $2 \times 10^{-2}\left(3 \times 10^{-3}\right)$. We also show that the HERA experiments cannot improve the present bounds on $\gamma_{B}$. Moreover, we demonstrate that the production at HERA and the Tevatron of di-jet events with large rapidity gaps between the jets cannot be explained by the existence of a light $\gamma_{B}$.
\end{abstract}

\section{INTRODUCTION}

Global symmetries of the standard model (SM), like baryon and lepton numbers, can be broken by quantum gravity effects [1]. In order to avoid an unacceptably large proton decay rate induced by this symmetry breaking we can elevate the conservation of baryon number to a local symmetry [2]. Moreover, there are string models that also gauge baryon number to protect the proton from decaying through higher-dimensional operators. In particular, models with low string scale, $E_{\text {string }} \sim \mathcal{O}(\mathrm{TeV})$, generally exhibit baryon number as a local symmetry [3].

In this work, we analyze the signals of a light gauge boson coupling to baryon number at the HERA and Tevatron colliders. We assumed that the $U(1)_{B}$ symmetry is spontaneously broken, giving a mass $\left(m_{B}\right)$ to $\gamma_{B}$, and that the mixing between $\gamma_{B}$ and the electroweak gauge bosons is negligible [4]. In the absence of mixing, the $\gamma_{B}$ boson always decays into quark pairs; its signature is thus the presence of 2 jets with an invariant mass close to $m_{B}$. A previous analysis [5] studied its effect on $Z$-pole physics and constrained its coupling to be $\alpha_{B} \lesssim 0.2$ for masses $m_{B} \leq m_{Z}$. Moreover, $\Upsilon(1 S)$ decays are modified by the $\gamma_{B}$ boson, leading to stronger constraints on $\alpha_{B}$ for $m_{B} \lesssim 30 \mathrm{GeV}$ [5].

In our analysis we concentrate on the decay $\gamma_{B} \rightarrow b \bar{b}$ in order to reduce the QCD backgrounds. Using a muon trigger, we show that the study of the production of $b \bar{b}$ pairs at the (upgraded) Tevatron can exclude $\gamma_{B}$ bosons with masses $\left(m_{B}\right)$ in the range $40 \lesssim m_{B} \lesssim 300 \mathrm{GeV}$ for couplings $\alpha_{B} \gtrsim 2 \times 10^{-2}\left(3 \times 10^{-3}\right)$. A displaced vertex trigger could increase the sensitivity at the upcoming Main Injector (MI) run by another factor of three. We also use the available search by the CDF collaboration for particles decaying into $b \bar{b}$ pairs [6]. Since this search uses a jet trigger which needs to be pre-scaled if the jet energies are not very large, the resulting limit turns out to be weaker than the sensitivity limit of current data based on a muon trigger. We also analyze $\gamma_{B}$ production at HERA, however, the potential bounds derived from this study are weaker than the $Z$-pole or Tevatron ones due to the limited integrated luminosity.

In principle, $\gamma_{B}$ boson exchange in the $t$-channel can give rise to events presenting a large rapidity gap between two jets 5 . Recently D $\varnothing$ studied the gap fraction as a function of $E_{T}$ [7]. Our fit to this data in terms of $\gamma_{B}$ exchange gives $\gamma_{B}$ masses very close to zero and $\alpha_{B} \simeq 0.06$. However, this region is already excluded by the analysis of $\Upsilon(1 S)$ decays [5]. Moreover, the HERA data on rapidity gaps can only be explained for $m_{B} \simeq 0$ and very large $\alpha_{B}$. Therefore, the present data on the production of rapidity gaps cannot be explained by the existence of a $\gamma_{B}$ boson.

In our analyses we assumed that the interaction between quarks and $\gamma_{B}$ is described by the effective Lagrangian

$$
\mathcal{L}_{\text {eff }}=\frac{1}{3} \sqrt{4 \pi \alpha_{B}} \bar{q} \gamma^{\mu} q B_{\mu},
$$

where we denote the quark fields by $q$ and the $\gamma_{B}$ field by $B_{\mu}$. Neglecting the fermions masses, the $\gamma_{B}$ width is given by

$$
\Gamma_{B}=\frac{N}{9} \alpha_{B} m_{B},
$$

where $N$ is the number of quark pairs to which $\gamma_{B}$ can decay.

\section{DIRECT SEARCHES AT TEVATRON AND HERA}

In hadronic collisions $\gamma_{B}$ bosons can be produced in the $s$-channel via quark-antiquark annihilation, leading 
to two-jet events. In order to reduce the large QCD backgrounds we focused on $\gamma_{B}$ decays into $b \bar{b}$ pairs $(p \bar{p} \rightarrow$ $\left.q \bar{q} \rightarrow \gamma_{B} \rightarrow b \bar{b}\right)$. The main backgrounds to this process are QCD $b \bar{b}$ production, mistagged QCD jets, and $Z$ production for $m_{B}$ close to $M_{Z}$. Since the two-jet event rate is too large for the present data acquisition system to be able to analyze all events, we need to choose a trigger to select a subset of the data. In our analyses of the Run I potential for $\gamma_{B}$ searches, we considered only events containing a muon (from $b$ decay) with $p_{T}^{\mu}>7.5 \mathrm{GeV}$ and $\left|\eta_{\mu}\right|<0.9$ (2 for the upgraded Tevatron) [8]. There is one useful byproduct of this requirement: together with the requirement that both $b$ 's be vertex tagged, the presence of this muon reduces the mistag background to a negligible level. Moreover, we also demanded that

- the jets should have $p_{T}>15 \mathrm{GeV}$;

- the jets should be separated by $\Delta R>0.4$.

With these requirements the $b$ tag efficiency is 0.24 . In our analyses we evaluated the scattering amplitudes of the signal and backgrounds using the package Madgraph [9], taking into account the interference between $\gamma_{B}$ and gluon $/ Z$ exchange diagrams.

We considered that a point of the $\left(\alpha_{B}, m_{B}\right)$ plane is within the reach of an experimental search, if the predicted signal has a $3 \sigma$ significance when we restrict ourselves to $b \bar{b}$ invariant masses in the range $m_{B}-10 \mathrm{GeV}<$ $m_{b \bar{b}}<m_{B}+10 \mathrm{GeV}$. We chose this rather high confidence level for an exclusion limit since our analysis does not allow for experimental resolutions or efficiencies (other than the $b$-tagging efficiency). We note, however, that CDF recently found [8] preliminary evidence for $Z \rightarrow b \bar{b}$ decays in their Run I data sample using cuts very similar to the ones applied by us. We show in Fig. 1 a the region in the $\left(\alpha_{B}, m_{B}\right)$ plane which could be excluded by the CDF Run I data, i.e. for an integrated luminosity of 110 $\mathrm{pb}^{-1}$ and a $b$-tagging efficiency of $24 \%$, if no signal is found. In this figure we also display the effect of having a larger integrated luminosity $\left(2 \mathrm{fb}^{-1}\right)$ and an extended rapidity acceptance for the muons $\left(\left|\eta_{\mu}\right|<2\right)$ at the MI. Notice that our $p_{T}$ and $\Delta R$ cuts constrain the invariant mass of the $b \bar{b}$ pair in two-jet events to be larger than 30 $\mathrm{GeV}$.

In order to explore smaller $\gamma_{B}$ masses we considered its production in association with a jet. The processes that we analyzed are

$$
\begin{aligned}
& p \bar{p} \rightarrow \gamma_{B} g \rightarrow b \bar{b} g, \\
& p \bar{p} \rightarrow \gamma_{B} q \rightarrow b \bar{b} q,
\end{aligned}
$$

where $q$ can be any quark or antiquark. We present in Fig. 1 $1 \mathrm{~b}$ the potential limits on $\alpha_{B}$ and $m_{B}$ originating from the study of $b-\bar{b}$-jet production for Run I and at the MI. In this analysis we applied the same cuts for the $b \bar{b}$ system and required the extra jet to have $p_{T}>10 \mathrm{GeV}$ and to be separated from the $b$ jets by $\Delta R>0.4$.
It is also possible to search for $\gamma_{B}$ using the Run I data but triggering on jets with a minimum $E_{T}[6]$. This choice of trigger requires pre-scaling, which leads to small effective integrated luminosities at low values of $E_{T}$. Using the CDF excluded production cross sections for particles decaying into $b \bar{b}$ pairs we obtained the limits on $\gamma_{B}$ shown in Fig. 2. We emphasize that the bounds shown in this figure are directly based on an experimental analysis, including all resolution and efficiency effects. As we can see, for $m_{B}$ up to a few hundred $\mathrm{GeV}$ the limits on $\gamma_{B}$ from this search are weaker than the ones that should be obtainable using the muon trigger, if no signal is found.

The Tevatron experimental collaborations are studying the possibility of triggering events exhibiting displaced vertices for the upcoming Main Injector run. We access the impact of this trigger on the searches for $\gamma_{B}$ eliminating the cuts on the muon coming from $b$ decays and introducing the QCD mistag background with a rate of $1 \%$ per jet. All other cuts are unchanged. Fig. 3 contains the region of the $\left(\alpha_{B}, m_{B}\right)$ plane that can be probed at the MI with this new trigger. As we can see from this figure, this trigger can increase the sensitivity of the Tevatron for $\gamma_{B}$ searches.

At HERA, $\gamma_{B}$ bosons can be produced via the hadronic content of the photon:

$$
\gamma p \rightarrow q \bar{q} \rightarrow \gamma_{B} \rightarrow q^{\prime} \overline{q^{\prime}}
$$

However, the two-jet signature of $\gamma_{B}$ is immersed in a large background from resolved photons. It turns out that the signal can not be observed even for the largest couplings allowed by the $Z$ physics $\left(\alpha_{B} \simeq 0.2\right)$ for the presently available luminosities. In order to observe a $\gamma_{B}$ signal for this large couplings one would need an integrated luminosity of at least $250-500 \mathrm{pb}^{-1}$ depending on $m_{B}$. Therefore, the bounds on $\gamma_{B}$ from HERA are much weaker than the $Z$ pole or Tevatron ones.

\section{RAPIDITY GAP ANALYSIS}

Experiments at HERA and the Tevatron have observed events containing two jets with no hadronic activity between them. These occur with a frequency of order of one percent in hadron-hadron collisions [10]. This is just one example of an interaction mediated by the exchange of the "Pomeron", a state which carries no net color. Since $\gamma_{B}$ is a color singlet, it can also give rise to rapidity gap events [5]. In this case the fraction of events presenting rapidity gaps as well as their kinematical distributions are determined by $\alpha_{B}$ and $m_{B}$, which allows us to constrain these parameters. Here we extract the bounds on $\gamma_{B}$ from the study of rapidity gaps assuming that these are only due to $\gamma_{B}$ exchange in the $t$-channel.

The D $\varnothing$ Collaboration has recently measured the production cross section of hard jets separated by a rapidity gap as a function of transverse momentum and gap size [7]. This data indicates that a large fraction of the 
gap events originates from quark-quark collisions [11, a feature that is present in the $\gamma_{B}$ exchange. In order to obtain bounds on $\gamma_{B}$ from this data, we evaluated

$$
F_{\text {gap }}\left(E_{T}\right)=\frac{d \sigma_{B} / d E_{T}}{d \sigma_{\text {total }} / d E_{T}},
$$

where $\sigma_{B}$ and $\sigma_{\text {total }}$ are the $\gamma_{B}$ contribution and the total cross section for the production of jet pairs, respectively.

In our analysis, we fixed the value of $m_{B}$ and determined $\alpha_{B}$ in order to fit the experimental $E_{T}$ spectrum, using the cuts and $E_{T}$ bins defined in Ref. [7]. We exhibit in Fig. 4 the region in the $\left(\alpha_{B}, m_{B}\right)$ plane obtained from the fits to the data. Although the $\chi^{2}$ distribution as a function of $\alpha_{B}$ has a well-defined minimum for all values of $m_{B}$, the quality of the fit is good only at small $m_{B}$. This can be seen from Fig. 5, which shows the general trend of the predicted $E_{T}$ distribution as $m_{B}$ increases. Therefore, $\gamma_{B}$ exchange cannot be the sole source of rapidity gap events at the Tevatron since the low mass allowed region in Fig. 1 has already been ruled out by the direct $\gamma_{B}$ search in $\Upsilon$ decays [5]. Furthermore, for larger $\gamma_{B}$ masses the fitted values of $\alpha_{B}$ fall well within the region that can be probed by the the direct search for $\gamma_{B}$ using Run I data, see Fig. 1. It is interesting to notice that introducing a gap survival probability 12 . $P_{s}<1$ only worsens the problem since this will require larger values of $\alpha_{B}$ to fit the data, $\alpha_{B} \propto 1 / \sqrt{P_{s}}$.

Hard jets separated by a rapidity gap have also been observed in photoproduction events at the ep collider HERA [13]. The ZEUS Collaboration measured that approximately $10 \%$ of the two-jet events with $p_{T}>6 \mathrm{GeV}$ and $\Delta \eta>3$ present a rapidity gap $\sqrt{13}$. Assuming that these events stem from $\gamma_{B}$ exchange in the $t$-channel we can constrain the mass and coupling of $\gamma_{B}$. We present in Table the values of $\alpha_{B}$ that lead to the observed gap fraction of 0.1 at large rapidity separation, where we imposed the cuts of Ref. [13]. Here it is also clear that these events cannot be explained solely as being due to $\gamma_{B}$ exchange.

\section{CONCLUSIONS}

In this work we demonstrated that the presently available Tevatron data can be used to rule out the existence of bosons coupling to baryon number for masses in the range $40-300 \mathrm{GeV}$ and $\alpha_{B} \gtrsim 0.02$, if no signal is found. In the near future, the Tevatron experiments should increase this sensitivity to $\alpha_{B} \gtrsim 0.003$ at the Main Injector. These bounds would preclude $\gamma_{B}$ boson exchange as a significant source of events with rapidity gaps between hard jets.

\section{ACKNOWLEDGMENTS}

We would like to thank T. Stelzer for helping us to introduce the $\gamma_{B}$ into the package Madgraph. This work was supported by Conselho Nacional de Desenvolvimento Científico e Tecnológico (CNPq), by Fundação de Amparo à Pesquisa do Estado de São Paulo (FAPESP), and by Programa de Apoio a Núcleos de Excelência (PRONEX).

[1] S. Giddings and A. Strominger, Nucl. Phys. B307 (1988) 854; S. Coleman, Nucl. Phys. B336 (1988) 643.

[2] H. Murayama and D. B. Kaplan, Phys. Lett. B336 (1994) 221; V. Ben-Hamo and Y. Nir, Phys. Lett. B339 (1994) 77; A. E. Faraggi, Nucl. Phys. B428 (1994) 111.

[3] G. Shiu and S.-H. Henry Tye, preprint CNLS 98/1561 (hep-th/9805157).

[4] For a model with a small mixing between $\gamma_{B}$ and electroweak bosons see C. D. Carone and H. Murayama, Phys. Rev. D52 (1995) 484.

[5] C. D. Carone and H. Murayama, Phys. Rev. Lett. 74 (1995) 3122.

[6] F. Abe et al., CDF Collaboration, hep-ex/9809022.

[7] J. Perkins (DØ Collaboration), Proceedings of the 5th International Workshop on Deep Inelastic Scattering and QCD, Chicago, Illinois, 1997; FERMILAB-Conf-97/250E. See also G. Snow, contribution to the Proceedings of the International Conference on High Energy Physics 1998.

[8] T. Dorigo for the CDF Collaboration, hep-ex/9806022.

[9] W. Long and T. Stelzer, Comput. Phys. Commun. 81 (1994) 357.

[10] S. Abachi et al. (DØ Collaboration), Phys. Rev. Lett. 72 (1994) 2332; F. Abe et al. (CDF Collaboration), Phys. Rev. Lett. 74 (1995) 855; Phys. Rev. Lett. 80 (1998) 1156; K. Goulianos (CDF Collaboration), Proceedings of the LAFEX International School on High Energy Physics (LISHEP-98), Rio de Janeiro, Brazil, 1998, FERMILABCONF-98/118-E.

[11] O. J. P. Éboli, E. M. Gregores, and F. Halzen, Phys. Rev. D58 (1998) 114005.

[12] J. D. Bjorken, Int. J. Mod. Phys. A7 (1992) 4189; Phys. Rev. D47 (1993) 101; preprint SLAC-PUB-5823 (1992).

[13] M. Derrick et al., ZEUS Collaboration, Phys. Lett. B369 (1996) 55; T. Ahmed, et al., H1 Collaboration, Nucl. Phys. B435 (1995) 3.

\begin{tabular}{||c|c||}
\hline \hline$m_{B}(\mathrm{GeV})$ & $\alpha_{B}$ \\
\hline \hline 10. & 1.05 \\
20. & 2.84 \\
30. & 4.59
\end{tabular}

TABLE I. Values of $\alpha_{B}$ needed to explain the formation of rapidity gaps in photoproduction events at HERA for several $\gamma_{B}$ masses. 

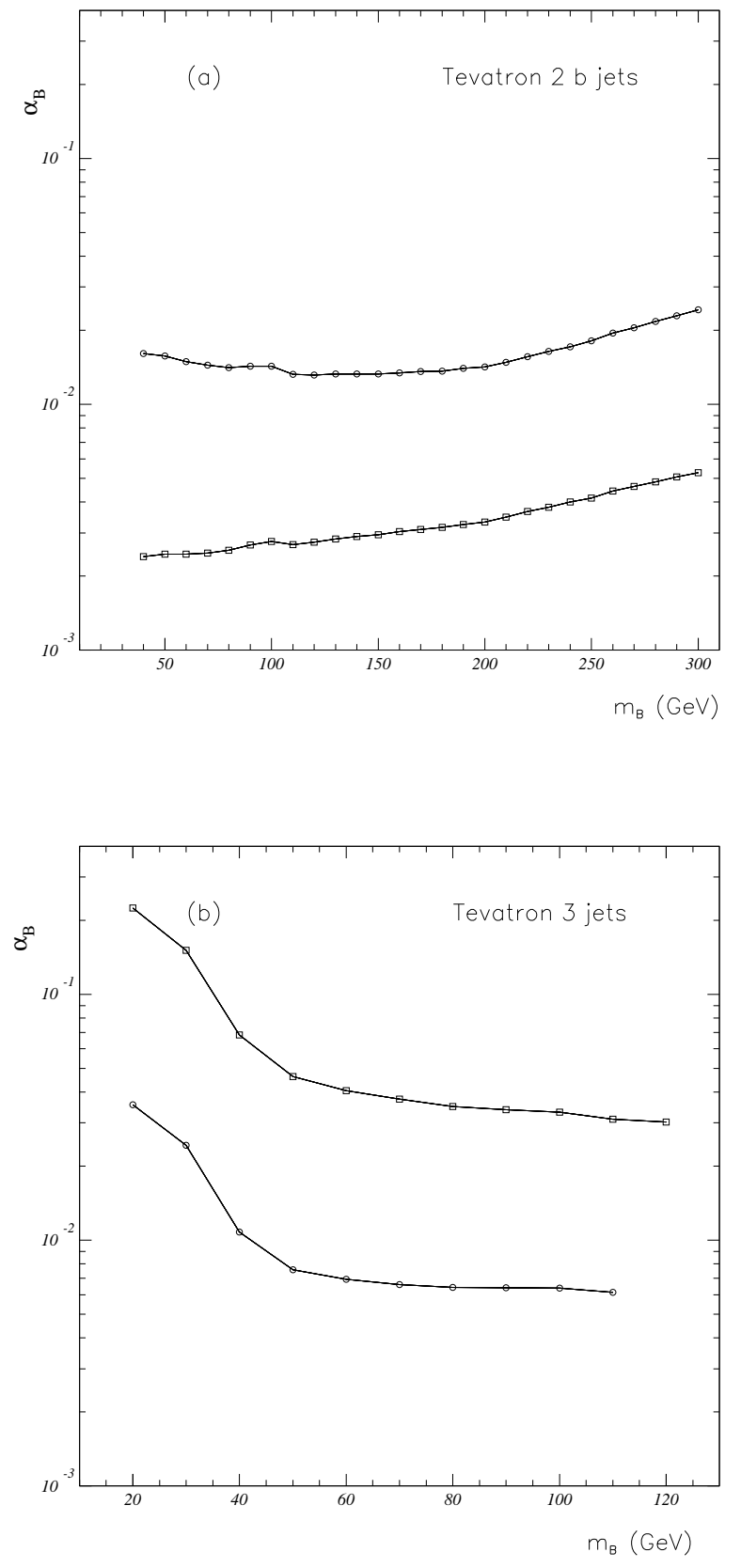

FIG. 1. The region that can be excluded at the $3 \sigma$ level using the Run I or Main Injector data is given above the upper and lower lines respectively. In (a) we considered $b \bar{b}$ final states while in (b) we studied $b \bar{b}+$ jet events.

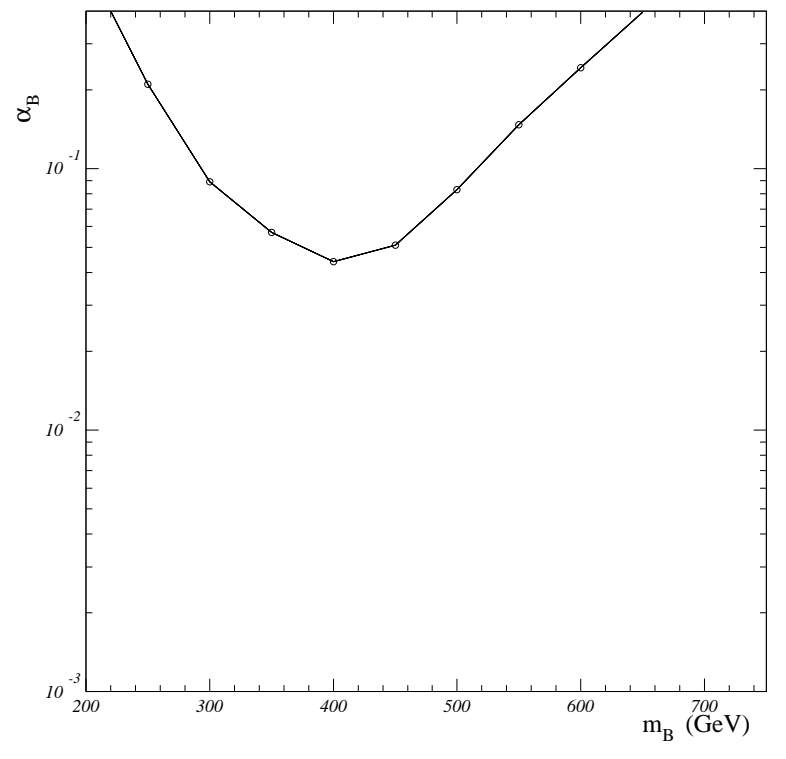

FIG. 2. The region that can be excluded at 95\% CL from the CDF search [6] using a jet trigger at Run I.

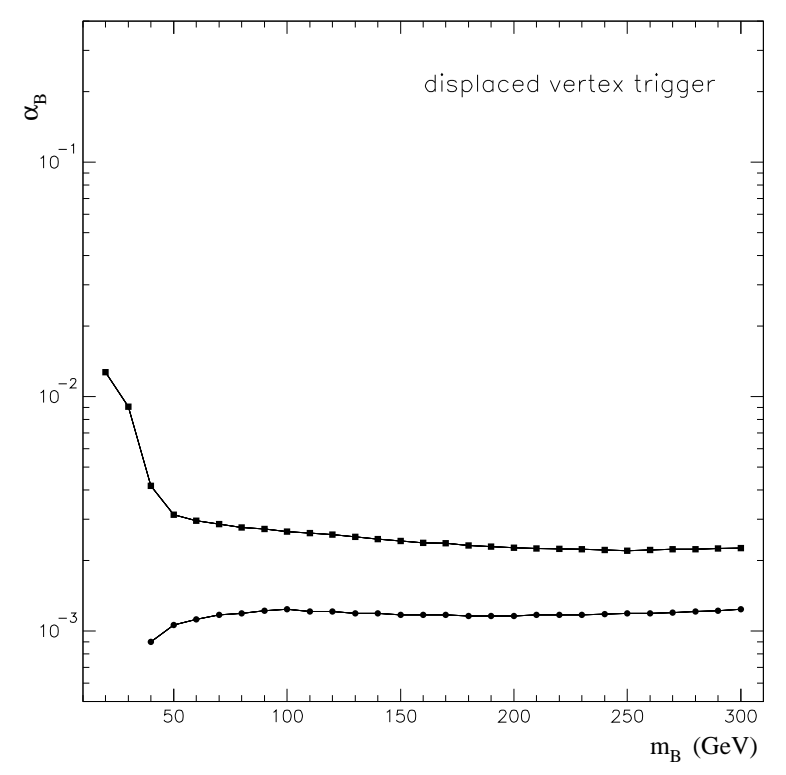

FIG. 3. The region that can be excluded at the $3 \sigma$ level at the MI using a displaced vertex trigger. The upper (lower) line is due to the $b-\bar{b}$-jet $(b \bar{b})$ production. 


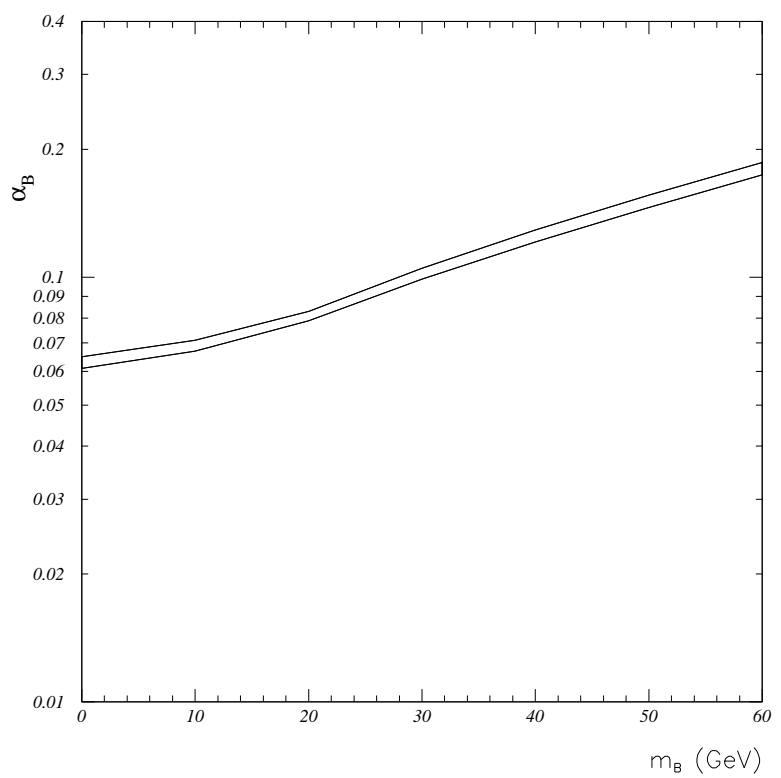

FIG. 4. The region between the solid lines is the $1 \sigma$ allowed area obtained from our fitting procedure to the $\mathrm{D} \varnothing$ data. We used the cuts and bins defined in Ref. [7].

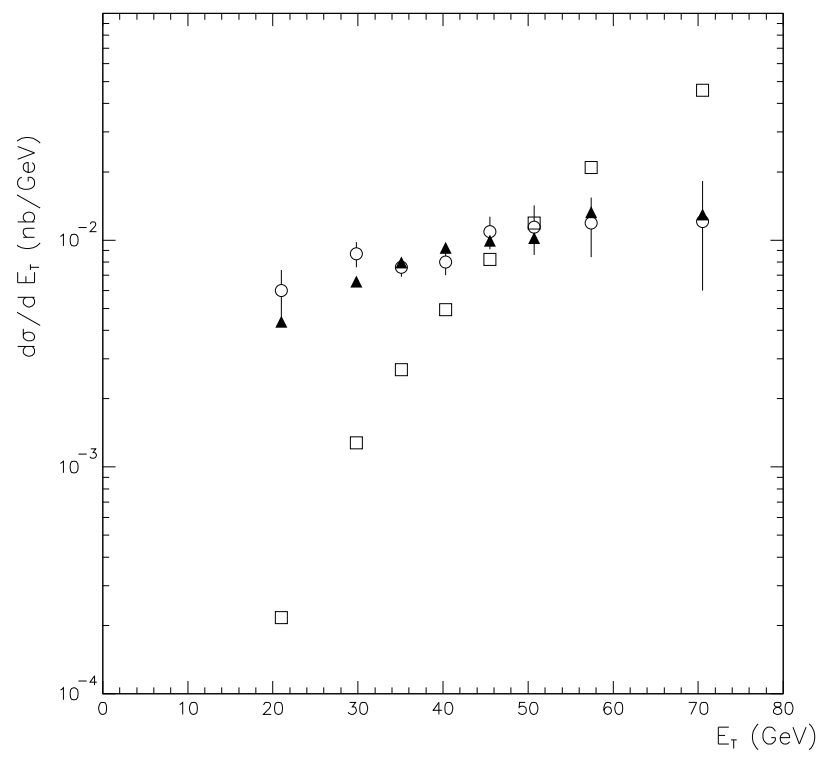

FIG. 5. Comparison of the experimental $E_{T}$ spectrum of the rapidity gap fraction (open circles) with the $\gamma_{B}$ predictions for $m_{b}=0$ and $70 \mathrm{GeV}$ (solid triangle and open squares, respectively). 